\title{
CRITERIOS CLÍNICOS Y RADIOLÓGICOS EN LA DECISIÓN TERAPÉUTICA DEL REFLUJO VESICOURETERAL. UN ANÁLISIS RETROSPECTIVO DE NUESTRA EXPERIENCIA
}

\author{
Armando Zuluaga Gómez, Mercedes Nogueras Ocaña, Francisco Valle Díaz de la Guardia, Víctor \\ López León, Antonio Jiménez Pacheco y Francisco Ramírez Garrido?.
}

Servicio de Urología. Servicio de Radiodiagnóstico'. Hospital Clínico Universitario San Cecilio. Granada. España.

\begin{abstract}
Resumen.- OBJETIVO: Establecer, en base a nuestra experiencia, unos esquemas terapéuticos del reflujo vesicoureteral en el niño, apoyándonos en resultados clínicos y radiológicos, observando cómo han ido evolucionando las indicaciones y tiempos de tratamiento con el advenimiento de las técnicas endoscópicas.
\end{abstract}

MÉTODOS: Se han analizado los pacientes con reflujo vesicoureteral tratados en dos periodos de similar duración: el primero (106 pacientes), entre 1995 y marzo 2001 (fecha en que se introdujeron las técnicas endoscópicas); el segundo, (138 pacientes), desde esa fecha hasta marzo de 2007. En todos los pacientes se estudiaron variables clínicas, diagnósticas y terapéuticas, así como el resultado obtenido.

RESULTADOS: El número de pacientes tratados aumentó en el segundo período, en él disminuyó ostensiblemente /de 24

\section{$\frac{0}{u}$
$\frac{1}{0}$
0
$\frac{1}{0}$
0
0
0
0
0}

Armando Zuluaga Gómez

Dr. Emilio Muñoz Fernández, 2 - 3ำ A

18012 Granada. (España).

azulvagagomez@hotmail.com a 7) el número de pacientes que requirieron reimplantación vesicoureteral. La proporción de éxitos del tratamiento endoscópico ascendió al 94,9\%, no apreciándose diferencias significativas en función de la edad o el grado de reflujo, aunque se observa un mayor porcentaje de fracasos en niños menores de 3 años y en reflujos de alto grado. La presencia de malformaciones asociadas no se relacionó con una peor evolución tras el tratamiento.

CONCLUSIONES: En aquellos reflujos donde el tratamiento médico no ha sido eficaz, persistiendo o empeorando el mismo, y como alternativa a la cirugía abierta (con buenos resultados pero con una morbilidad no desdeñable), el tratamiento endoscópico se convierte en una alternativa de eficacia similar y mínimamente agresiva. Proponemos un esquema orientativo para establecer las indicaciones de cada estrategia terapéutica en función del grado de reflujo y su evolución clínica.

Palabras clave: Reflujo vesicoureteral. Tratamiento. Pediatría.

Summary.- OBJECTIVES: According to our ex-perience, we present a proposal for the treatment of vesicoureteral reflux, based on both clinical and radiolo-gical evidences. We also describe how the introduction of endoscopic procedures has influenced the evolution of treatment indications as well as the time intervals for treatment.

METHODS: We have analysed all cases of vesicourete-ral reflux treated in our Department in two periods of similar length: The first one (106 patients) comprised from 1995 to March 2001 (when endoscopic procedures were introduced). The second one (138 patients), comprised from March, 2001 to March 2007. Clini-cal, diagnostic, therapeutic and outcomerelated varia-bles were studied for all cases.

RESULTS: The number of patients was higher in the se-cond period. In this period the number of cases requiring ureteral 
reimplantation decreased with respect to the first one (from 24 to 7). The success rate with en-doscopic treatment reached $94,9 \%$, with no significant differences regarding age or grade of reflux, although higher rates of failures were observed in children aged less than 3 years old and in high-grade reflux. The asso-ciation of reflux with other malformations was not related with a worse evolution after treatment.

CONCLUSIONS: Endoscopic treatment, due to its similar efficacy and low aggressiveness, should be con-sidered a valid alternative to open surgery (which offers good results but non-negligible comorbidity) for persistent reflux in which medical treatment has not been useful. We propose a tentative therapeutic scheme to establish the indications for each type of treatment depending on the grade of reflux and its clinical evolution.

Keywords: Vesicoureteral reflux. Treatment. Pediatrics.

\section{INTRODUCCIÓN}

El reflujo vesicoureteral en la infancia representa una patología relativamente frecuente, con una incidencia del $1 \%$ en niños sanos, siendo de gran trascendencia por las consecuencias que, si no se diagnostica y trata adecuadamente, puede suponer por su repercusión en tres aspectos: crecimiento físico, hipertensión y potencial insuficiencia renal crónica. El reflujo vesicoureteral está o ha estado presente en un 30 a un $40 \%$ de los niños que tienen insuficiencia renal antes de los 16 años y en un $20 \%$ de los adultos que tienen insuficiencia renal antes de los 50 años (1).

La presencia de una infección urinaria en recién nacidos y lactantes puede llevarnos al diagnóstico de reflujo vesicoureteral hasta en un 50\% de los casos; algunos podrán ser tratados médicamente y otros requerirán tratamiento quirúrgico, dependiendo de una serie de variables clínicas, radiológicas, funcionales $y$, en algunos casos, endoscópicas. Este reflujo puede desaparecer espontáneamente, aunque esto es más fácil que suceda en los primeros años de vida, más difícil a partir de los 10 años y prácticamente imposible en los adultos (2).

Aunque los conocimientos acerca de la fisiopatología del reflujo vesicoureteral, su clínica y sus métodos diagnósticos, han variado poco con el correr del tiempo, no puede decirse lo mismo de las estrategias terapéuticas. Esencialmente, los objetivos del tratamiento del reflujo vesicoureteral son claros y evidentes: 1- Desaparición del reflujo, 2- Desaparición y control de la infección urinaria, 3- Evitar cuadros de pielonefritis aguda, y 4- Evitar la aparición de cicatrices renales y daño parenquimatoso e insuficiencia renal crónica (3). Para conseguir estos objetivos nos apoyamos en tres pilares, dependiendo de la clínica (infecciones urinarias complicadas o no complicadas), los hallazgos radiológicos (grado del reflujo), la analítica (cultivos de orina) y las pruebas de funcionalismo renal. Estos tres pilares son: a) Observación y seguimiento; b) Tratamiento médico; c) Tratamiento quirúrgico.
Con respecto a la evolución del tratamiento del reflujo vesicoureteral, al margen de la mejora de los tratamientos médicos, con la aparición de nuevos fármacos, más efectivos y de mejor manejo en el tratamiento de las infecciones urinarias, es sobre todo destacable el avance de los tratamientos quirúrgicos. En general, se puede diferenciar claramente un primer periodo donde se consideraba la cirugía como la primera opción, seguido por otro, en la década de los 80 , en el que disminuyeron sus indicaciones a favor de los tratamientos médicos, hasta la actualidad, en donde las técnicas endoscópicas, difundidas por Puri (4), han pasado a ocupar un papel preferente como primera alternativa en muchos grados de reflujo, con resultados altamente satisfactorios. Puesto que, en mayor o menor medida, nos ha tocado vivir cada una de estas épocas, creemos que estamos en condiciones de emitir un juicio razonado al respecto, basado en nuestra propia experiencia, aunque dejando claro que no se puede dogmatizar en un terreno en donde en muchísimas ocasiones cada caso puede tener enfoques diferentes. Por ello, el objetivo del presente trabajo es establecer, apoyados por el análisis retrospectivo de nuestra casuística, unos esquemas terapéuticos del reflujo vesicoureteral en el niño basados en resultados clínicos y radiológicos, observando cómo han ido evolucionando las indicaciones y los tiempos de tratamiento con el advenimiento de las técnicas endoscópicas.

\section{MATERIAL Y MÉTODOS}

Hemos revisado los pacientes con reflujo vesicoureteral primario remitidos por el Servicio de Pediatría - diagnosticados directamente en nuestra Unidad de Urología Pediátrica. Para su estudio consideramos dos períodos: uno, desde el momento en que introdujimos el tratamiento endoscópico en nuestros pacientes (marzo de 2001), hasta marzo del 2007; otro, desde ese momento hacia atrás, hasta marzo de 1995. En estos dos periodos de igual duración (6 años) hemos considerado las siguientes variables:

\section{1). Sexo y edad ( $\leq 3$ años $y>3$ años).}

2). Motivo del diagnóstico (casual, infección urinaria +/fiebre, diagnóstico prenatal o presencia de dilatación pielocalicial ecográfica),

3). Variables obtenidas de las pruebas diagnósticas:

3.1. CUMS, realizada con solución contrastada a $37^{\circ}$ con placas AP y oblicuas, en fase de llenado y miccional. A partir de ella se valoró la localización del reflujo (derecho, izquierdo o bilateral) y el grado del mismo (I a V, según la Figura 1).

3.2. Ecografía: Esta prueba permite valorar si existe alteración del contorno renal (lobulaciones, áreas cicatriciales), reducción del grosor parenquimatoso, y la existencia o no de dilatación de la vía excretora;

3.3. Pruebas isotópicas (DMSA, MAG-3): Para determinar la existencia de alteraciones de la función de la unidad renal refluyente y la presencia o no de cicatrices renales. 
4). Variables del Tratamiento: En este apartado se han considerado los tres tratamientos empleados:

4.1. Tratamiento médico: mediante la administración de una quimioprofilaxis nocturna a un tercio de la dosis, de distintos antibióticos según antibiograma lamoxicilina, trimetropim-sulfametoxazol, fosfomicina o cefuroxima-acetilo). Se realizaron controles con urocultivos mensuales, ecografías semestrales y nueva CUMS al año. Se valoró el tiempo de evolución desde el diagnóstico y la aplicación del tratamiento médico hasta la curación o cambio a otro tratamiento (endoscópico o quirúrgico abierto), así como los motivos que llevaron a ese cambio: aparición de pielonefritis, cicatrices renales o persistencia del reflujo sin reducción del grado. En lactantes con reflujo de alto grado o bilateralidad, se indicó tratamiento médico de inicio pero se adelantó el control de la CUMS a los 6-9 meses.
4.2. Tratamiento quirúrgico: la técnica aplicada ha sido la de Leadbetter-Politano en la mayoría de los casos unilaterales, y la técnica de Cohen en todos los bilaterales y algunos unilaterales. Se hizo control postoperatorio con ecografía al mes y CUMS a los 6 meses.

4.3. Tratamiento endoscópico: siguiendo la técnica descrita por Puri y O’Donnell, mediante la inyección endoscópica submeatal de distintas sustancias biocompatibles: Macroplástique ${ }^{\circledR}$ (polímero bifásico de polydimethylesiloxane en un hidrogel), Deflux ${ }^{\circledR}$ (dextranómero del copolímero del ácido hialurónico) y Coaptite ${ }^{\circledR}$ (hidroxiapatita). La intervención se realiza bajo sedación y en hospital de día. Se llevó a cabo un control postoperatorio similar al de la cirugía abierta: ecografía al mes, CUMS a los 6 meses y, en algunos casos, ecosonocistografía.

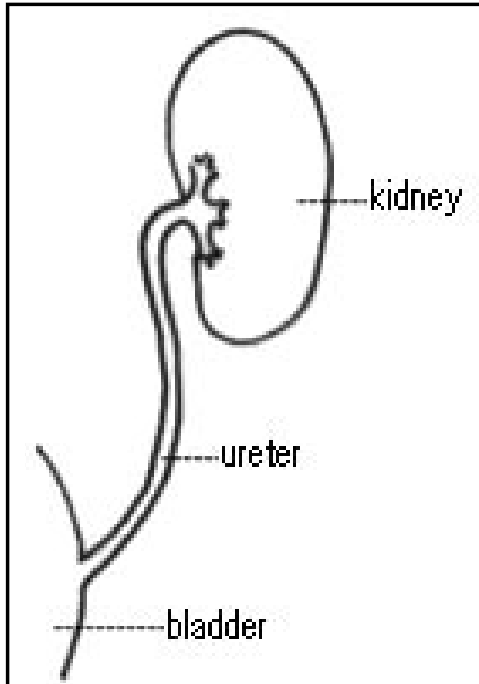

Riñón, uréter y vejiga normales.

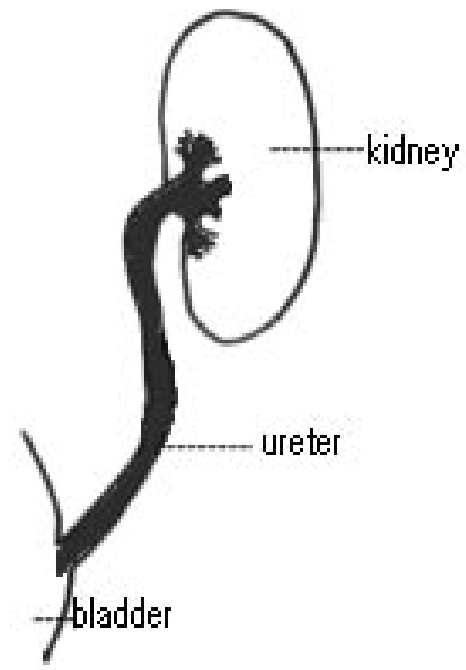

Reflujo vesicoureteral grado III.

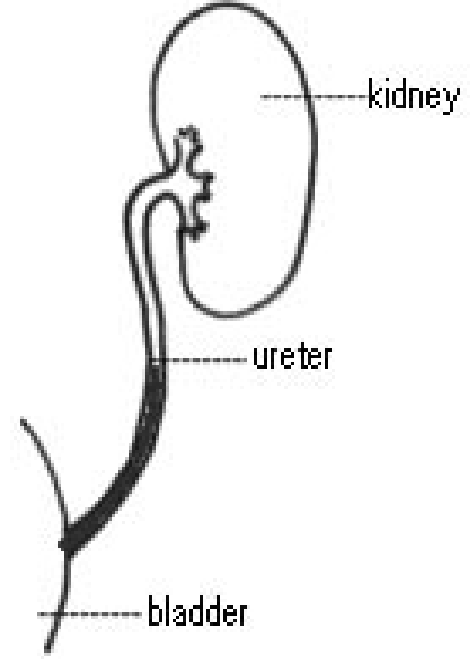

Reflujo vesicocureteral grado I.

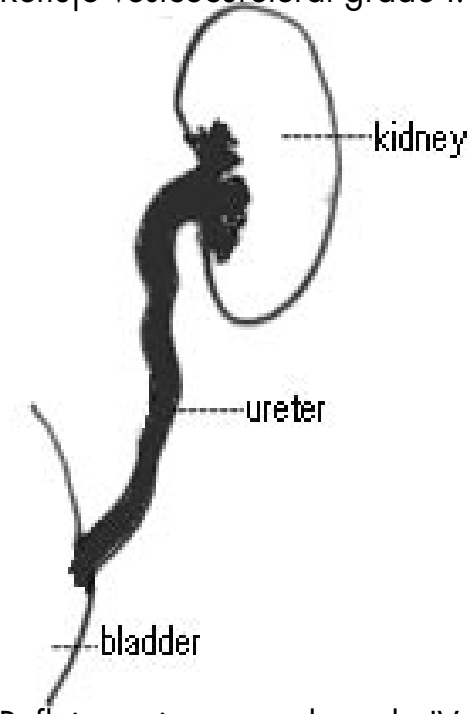

Reflujo vesicoureteral grado IV.

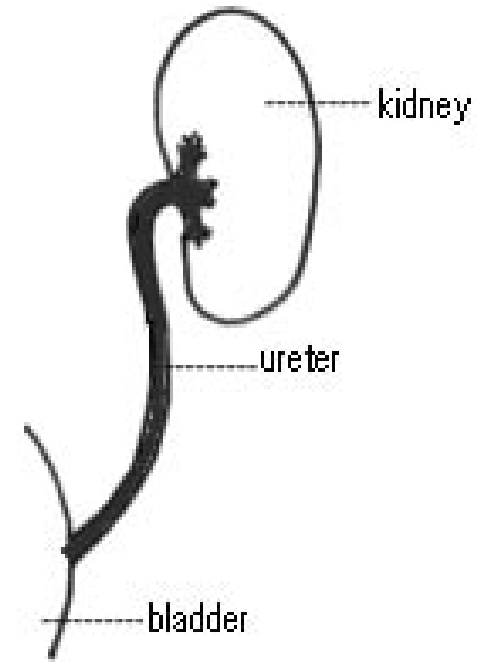

Reflujo vesicoureteral grado II.

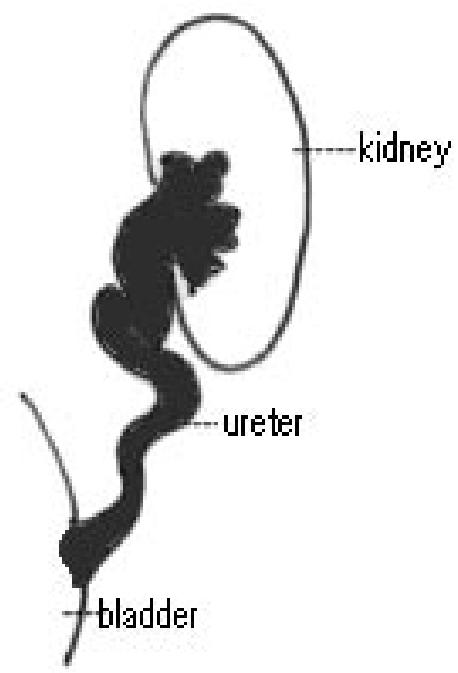

Reflujo vesicoureteral grado $\mathrm{V}$ cortical parcial o total. 
5). Valoración de los resultados: curación o persistencia del reflujo con nuevo tratamiento endoscópico. En los casos en los que se realizó estudio urodinámico se determinó la presencia de factores que influyeran en el fracaso terapéutico.

Todas las variables antes enumeradas se estudiaron separadamente en cada uno de los dos grupos de pacientes definidos previamente. Se ha utilizado el test Chi2 para comparar la distribución de variables categóricas.

\section{RESULTADOS}

En el periodo completo estudiado (1995-2006), hemos tratado un total de 246 pacientes diagnosticados de reflujo vesicoureteral. De ellos, 106 corresponden al primer periodo (1995-2000) y 138 al segundo. La distribución de los tratamientos en función del período considerado se muestra en la Figura 2.

En los primeros 106 pacientes se llegó al diagnóstico tras la aparición de una infección urinaria +/- fiebre en el $87,5 \%$ de los casos; en el resto fue de forma casual - por un diagnóstico prenatal de dilatación renal. A 96 pacientes se les aplicó un tratamiento médico con quimioprofilaxis nocturna; 59 eran niños y 37 niñas. De ellos, 14 pasaron a tratamiento quirúrgico tras un periodo de tratamiento médico que osciló entre el año y los 4 años, con una media de 1,2 años. Otros 10 niños se operaron directamente tras realizarse el diagnóstico de reflujo, sin esperar a probar con tratamiento médico. En total se intervinieron quirúrgicamente en este periodo 24 niños, 11 varones y 13 niñas, con edades comprendidas entre los 2 meses y los 15 años (media de 4,4); 8 niños se operaron por debajo del año de edad, 5 entre el año y los 3 años y el resto, 11 casos, con más de 3 años. La localización del reflujo fue derecha en 4 casos, izquierda en 12 y bilateral en 8 . Con respecto al grado del reflujo, 7 fueron grado II, 12 grado III, 9 grado IV y 4 grado V. El motivo que llevó a

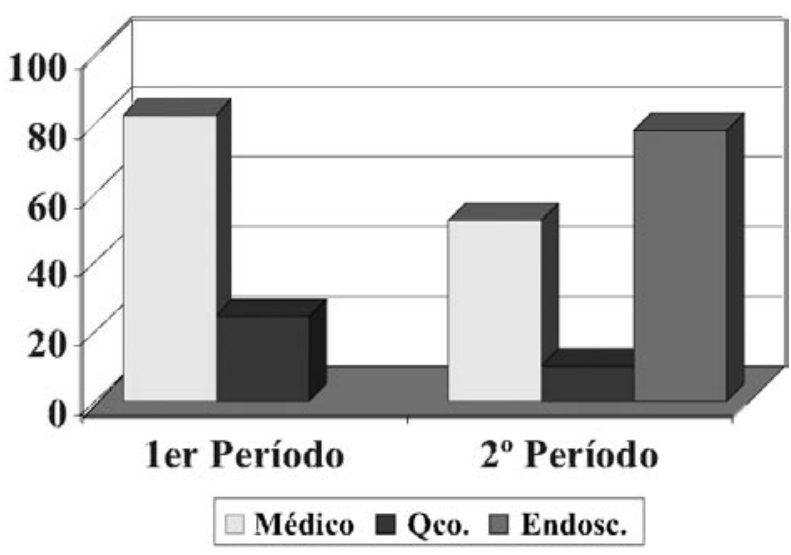

FIGURA 2. Distribución de los tratamientos en función del periodo considerado. decidir este tratamiento fue principalmente la aparición de un episodio de pielonefritis, lo que ocurrió en el $58,3 \%$ de los casos; en el resto se debió a la no resolución del reflujo con el tratamiento médico. En la ecografía realizada al grupo de pacientes operados se encontraron alteraciones en 21 casos, predominando la dilatación pielocalicial; las pruebas isotópicas demostraron alteraciones (disminución de la FRR o aparición de cicatrices renales) en el 71,4\% de los casos. La técnica quirúrgica aplicada fue la $\mathrm{T}$. de Cohen en 14 niños $(58,3 \%)$ y la de Leadbetter-Politano en el $41,7 \%$ restante. Cinco casos tenían malformaciones asociadas: doble sistema, megavejiga o divertículo vesical. Todos los casos evolucionaron sin complicaciones postoperatorias, salvo uno que requirió transfusión sanguínea. En todos los pacientes se consiguió la resolución del reflujo comprobada en la CUMS realizada a los 6 meses tras la intervención, quedando sólo en un caso un reflujo residual grado I.

En el segundo periodo tratamos un total de 138 niños: 52 mediante tratamiento médico, 78 con tratamiento endoscópico y 10 con tratamiento quirúrgico abierto, debiéndose constatar que a 3 de estos últimos pacientes se les realizó nefroureterectomía por anulación funcional de la unidad refluyente. Los pacientes que se han resuelto o que están actualmente con tratamiento médico son 17 niños y 35 niñas, de edades comprendidas entre los 10 meses y los 5 años (media de 2,4).

El tratamiento endoscópico se aplicó a 78 niños con edades entre los 4 meses y los 18 años (media de 5,4), $66,7 \%$ de ellos de sexo femenino. La localización del reflujo fue derecha en 21 casos, izquierda en 32 y bilateral en 25. La distribución de los grados fue la siguiente: I, 6,4\%; II, 20,5\%; III, 21,8\%; y IV, 13,6\%. El motivo que llevó al tratamiento endoscópico fue, en 19 casos, episodios de ITU repetidas o pielonefritis; en 57 casos $(73,1 \%)$, la no resolución del reflujo a pesar de la quimioprofilaxis; en los dos casos restantes se dieron ambas causas.

El tiempo transcurrido entre el diagnóstico y el tratamiento endoscópico osciló entre el mes y los 6 años, con una media de 1,6 años. Para el tratamiento se empleó polidimetilsiloxano (Macroplástique $®$ ) en 32 casos, en 45 microesferas de dextrano (Deflux $®$ ) y en un caso hidroxiapatita (Coaptite $($ ). La evolución postoperatoria (Figura 3) fue favorable, con resolución del reflujo tras la 1 in inyección en el $71,8 \%$; en 5 casos se puso de manifiesto un reflujo contralateral que se trató con buen resultado (Figuras 4-6). A esto hay que añadir 4 casos $(5,1 \%)$ en los que persistió un reflujo grado I activo y que están actualmente en observación. En los 18 casos restantes $(23,1 \%)$ fue necesaria una $2^{\mathrm{a}}$ inyección, resolviéndose 14 de ellos, lo que eleva el resultado de éxitos al 94,9\%; en los 4 casos en los que ha persistido el reflujo, 2 están pendientes de una $3^{\underline{a}}$ inyección siendo reflujos grado II unilaterales; un caso requirió tratamiento quirúrgico abierto por tratarse de un grado III-IV monorreno. En cuanto al caso restante, tras fracasar la segunda inyección y haber aumentado el grado del reflujo, se realizó un estudio urodinámico, que reveló una vejiga hiperactiva, por lo que requirió tratamiento con toxina botulínica, al no haber sido efectivo el uso de anticolinérgicos. 
No se ha presentado ninguna complicación importante durante el postoperatorio, salvo 4 casos de infección urinaria y 2 de hematuria que cedió espontáneamente. El estudio urodinámico se realizó en 6 casos en los que falló la primera inyección; en 4 de ellos se asoció tratamiento con anticolinérgicos. Este tratamiento también lo hemos pautado de entrada a 23 pacientes $(29,5 \%)$, que tenían algún síntoma de irritabilidad vesical. En 16 casos existía alguna malformación asociada, destacando 4 casos de doble sistema, 2 monorrenos, 2 divertículos paraureterales (Figuras 7 y 8) y 2 megavejigas, lo que no influyó en el resultado final.

No se observan diferencias estadísticamente significativas al comparar los resultados del tratamiento endoscópico en función de los grupos de edad $1 \leq 3$ y $>3$ años). (Figura 9) Tampoco las hubo con respecto al grado del reflujo (Figura 10), aunque puede apreciarse un mayor porcentaje de éxitos en los reflujos de bajo grado. Finalmente, si se apreciaron diferencias en cuanto al sexo, pues la proporción de éxitos fue significativamente superior en las niñas $(84,6 \%$ vs $61,5 \%$ en niños, $p=0,046)$.

Por último, en este segundo periodo sólo hemos operado mediante cirugía abierta a 10 niños, de los que 3 precisaron una nefroureterectomía por anulación funcional de la unidad renal, en todos ellos con reflujo grado V. En 4 niñas y 3 niños realizamos reimplantación; en 6 casos, con edades por debajo del año y medio, la indicación se debió a un cuadro infeccioso; en el caso restante (un niño de 6 años monorreno) se debió a 2 fracasos previos de cirugía endoscópica. La localización del reflujo en estos 7 pacientes fue derecho en 1 caso, izquierdo en 3 y bilateral en los restantes. El grado del reflujo en las 10 unidades renales reimplantadas ( 3 según T. de Leadbetter y 6 con T. de Cohen) fue II en 3 casos, III en 1 caso, IV en 5 casos y $\mathrm{V}$ en 4 casos. Todos estos pacientes presentaban dilatación pielocalicial en la ecografía y alteraciones funcionales en el DMSA, con cicatrices renales en uno de ellos. Dos pacientes eran monorrenos y un tercero tenía asociado un divertículo paraureteral. Los resultados de este subgrupo han sido satisfactorios, con resolución del reflujo en todos

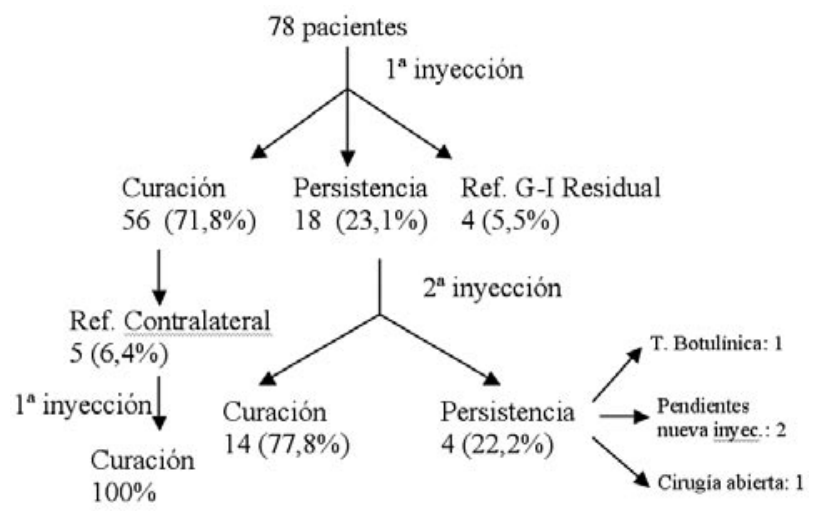

FIGURA 3. Evolución de los pacientes tras tratamiento endoscópico. los casos; en dos de ellos persiste cierta dilatación piélica residual en la ecografía con buena eliminación renal en el renograma.

\section{DISCUSIÓN}

Es muy difícil, por no decir imposible, establecer un protocolo estanco para decidir el tratamiento de cualquier patología. El número de variables que se pueden considerar es tan amplio que harían imposible basar la decisión terapéutica sobre este ingente número de posibilidades. No obstante, los esfuerzos deben ir encaminados a simplificar $y$, sobre la base de variables muy concretas, basar la decisión a tomar.

Los clásicos estudios de Dwoskin y Perlmutter (5), Heikel y Pakkulainen (6) y del Grupo Internacional de Estudio del Reflujo (7) que clasifican los reflujos dependiendo de las imágenes radiológicas obtenidas mediante cistografía retrógrada, han contribuido muy positivamente en la decisión terapéutica.

Para los controles evolutivos del reflujo se ha empleado tradicionalmente la CUMS mediante contraste yodado, aunque en base al desarrollo de los medios de

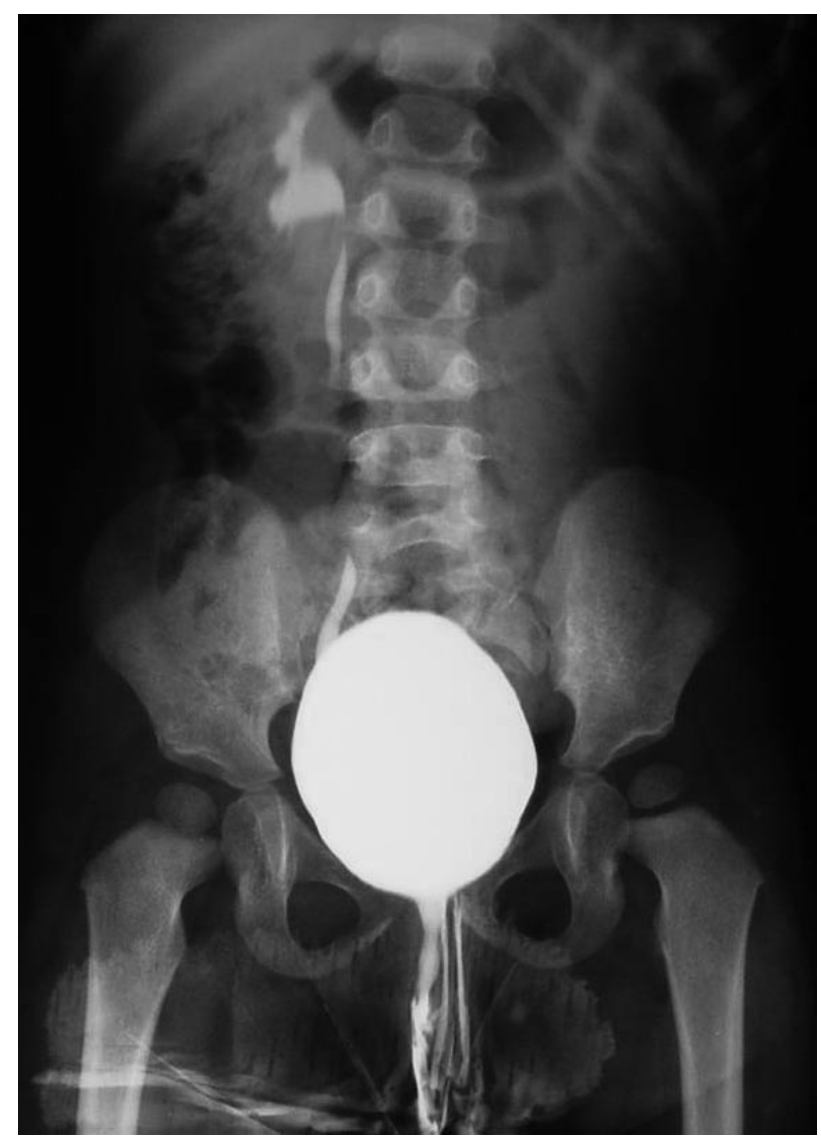

FIGURA 4. Caso 1a. Reflujo vesicoureteral derecho. 


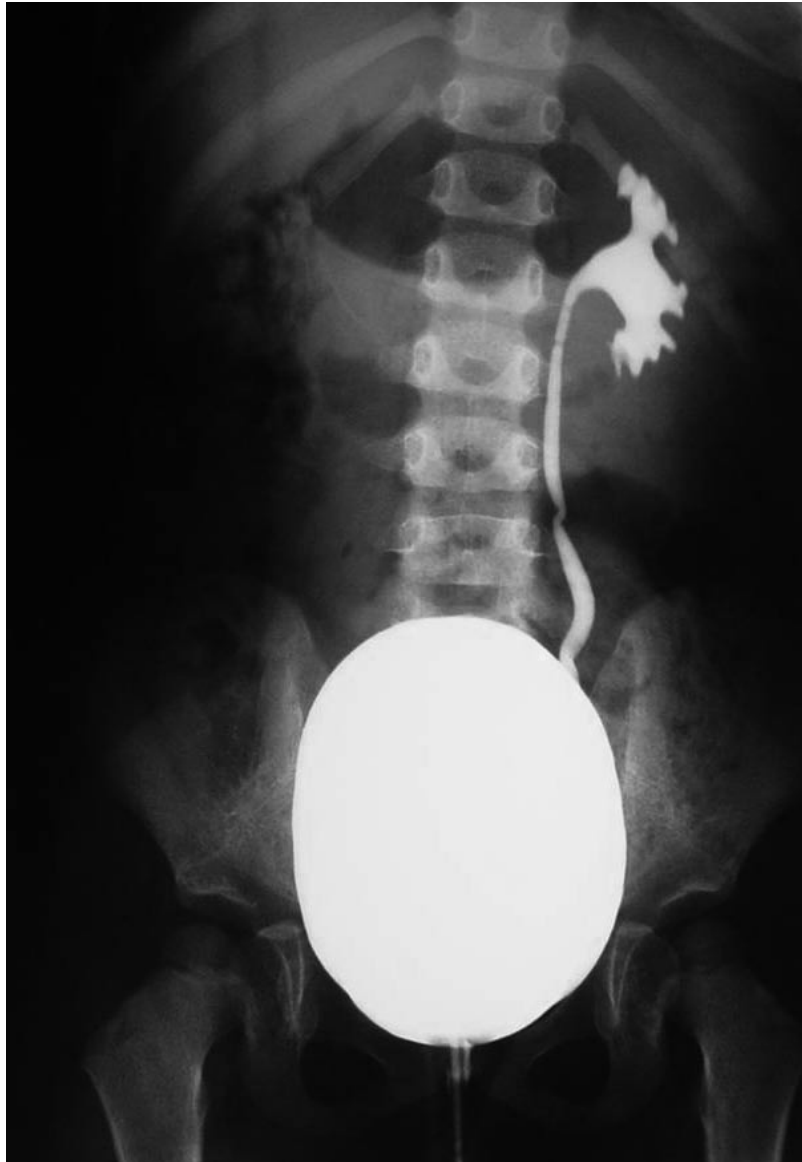

FIGURA 5. Caso 1b. Tratamiento endoscópico derecho: curación y aparición de reflujo contralateral.

contraste ecográfico de $2^{\underline{a}}$ generación, como el Levograf $\circledast$ (suspensión de galactosa), la ecosonocistografía constituye un método fiable y seguro, exento de radiación, cuyo uso debería potenciarse, ya que no parecen existir diferencias significativas a la hora de detectar y cuantificar el reflujo con respecto a la cistografía convencional. Según algunos autores $(8,9)$, la concordancia de ambas pruebas ronda el $90 \%$

Lyon (10), en 1969, introdujo una clasificación sobre la morfología del orificio ureteral basada en la visión endoscópica que, según el autor, constituiría un dato más a tener en cuenta en el tratamiento a aplicar. Así, distingue cuatro grupos morfológicos: a)- Orificio en volcán, b)- en estadio, c)- en herradura y d)- en hoyo de golf (fig 4). Los dos primeros no serían refluyentes y si lo fuesen en algún momento, el reflujo fácilmente desaparecería de forma espontánea. Los dos últimos serían claramente refluyentes, fundamentalmente el último, en donde un tratamiento quirúrgico más o menos invasivo sería necesario para su corrección. Lyon relaciona claramente esta morfología orificial con defectos anatómicos del uréter intramural tanto en longitud, oblicuidad, lateralidad y desarrollo muscular.

La presencia de infección urinaria única o recidivante ha sido uno de los parámetros clínicos fundamentales

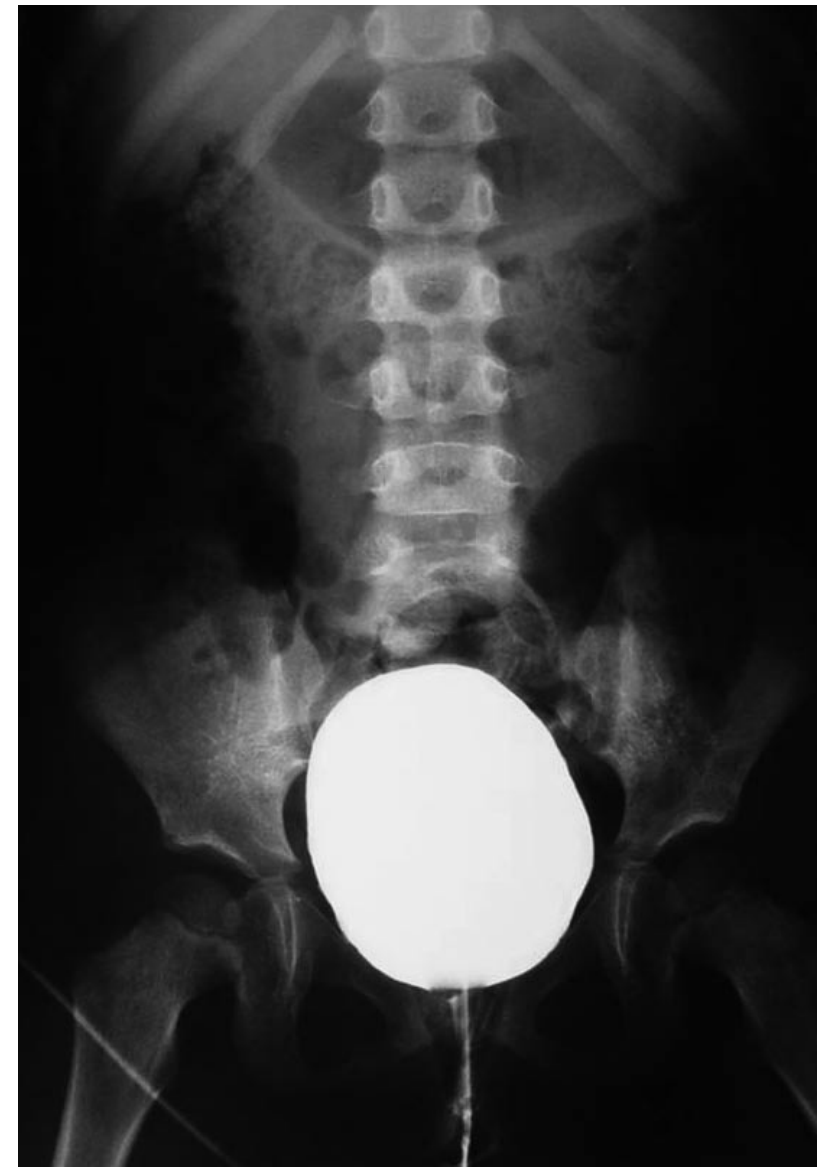

FIGURA 6. Caso 1c. Nuevo tratamiento endoscópico izquierdo y curación definitiva.

para decidir el tratamiento médico o quirúrgico a emplear. Cuando ésta es una infección no complicada, el tratamiento profiláctico de larga duración tiene aquí su principal indicación. En la mayoría de los casos de reflujo grado I y II y en algunos de grado III se pueden conseguir controles óptimos que, además, a la larga consiguen hacer desaparecer el reflujo, gracias entre otros motivos a la normalidad anatómica de la unión ureterovesical, en donde el uréter intramural conserva su oblicuidad, longitud y conformación muscular normal.

Cuando la infección urinaria es complicada y se acompaña de cuadros febriles, con bacteriemia e incluso con grave repercusión general, es evidente y muchos autores lo afirman (11), que debe establecerse un tratamiento más activo médico-quirúrgico. Estos cuadros sintomáticos aparecen en ciertos reflujos grado III y casi siempre en los reflujos grado IV y V, en donde además se sobreañaden lesiones parenquimatosas que poco a poco deterioran el funcionalismo renal y que, cuando son bilaterales, llevan a estos niños a la insuficiencia renal ya en edades tempranas, siendo motivo de diálisis y trasplante por nefropatía de reflujo (3).

Se ha puesto de manifiesto, primero en estudios experimentales en cerdos y posteriormente en humanos, la 


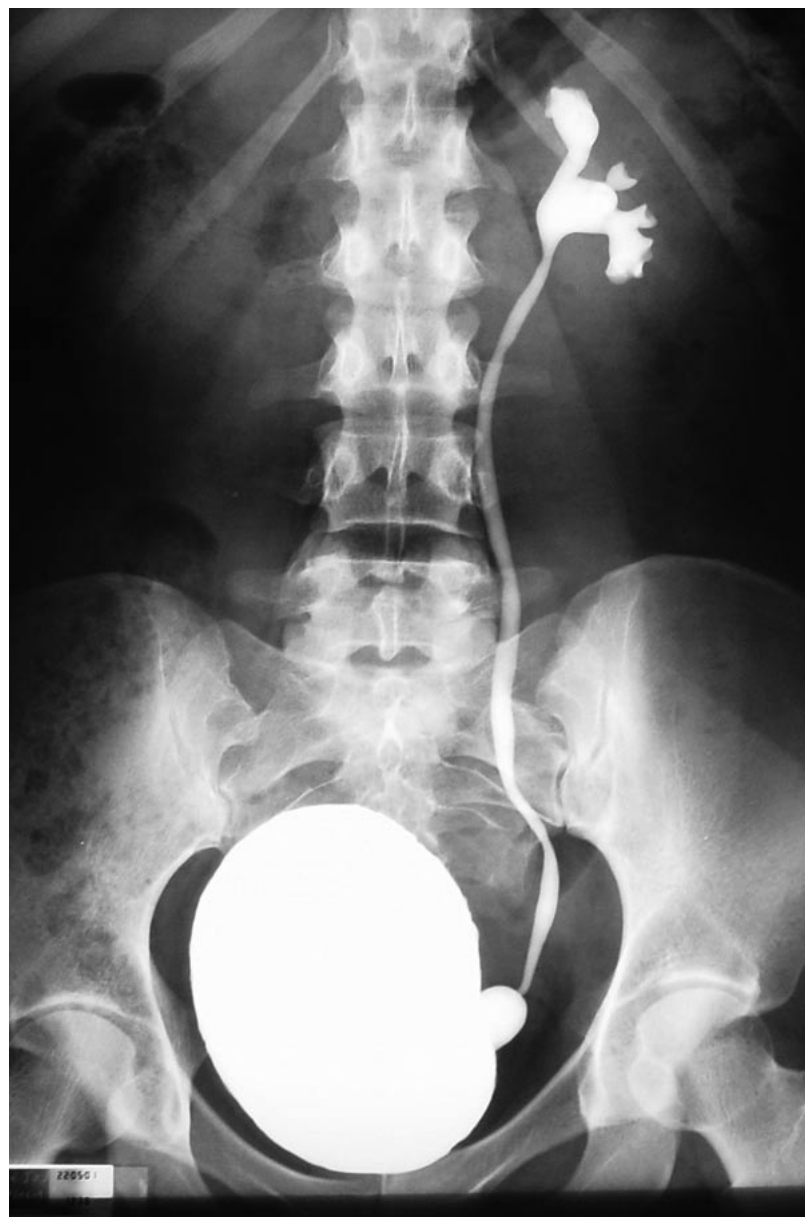

FIGURA 7. Caso 2a. Reflujo asociado a divertículo.

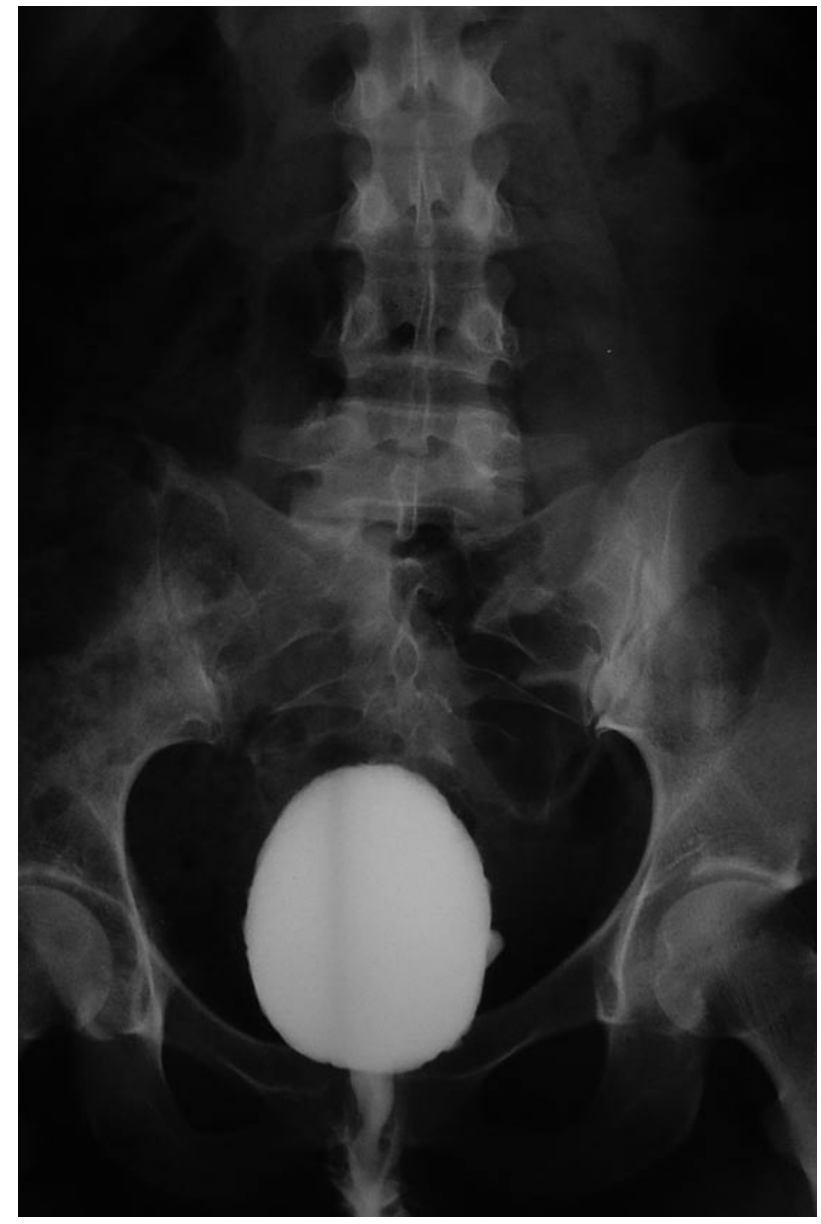

FIGURA 8. Caso 2b. Tratamiento endoscópico y curación.

se consigan los mayores beneficios con el menor riesgo de daño renal y la menor morbilidad con la técnica empleada (11). Así, aunque el reimplante ureteral mediante cirugía abierta alcanza en la mayoría de las series un éxito del prácticamente $95 \%$, su indicación se suele reservar para aquellos casos donde la severidad del reflujo compense el riesgo quirúrgico (15).

Parece existir consenso $(3,11,16)$ en aplicar un tratamiento médico inicial a todos los reflujos grado I y II, dada la alta probabilidad de desaparición espontánea; los grados III y IV son para muchos también subsidiarios de comenzar con un tratamiento conservador. En nuestro caso así lo hemos hecho, consiguiendo algunas veces la desaparición del reflujo y otras la reducción del grado. Otros autores, sin embargo, avalan un tratamiento endoscópico preferente para estos grados con objeto de eliminar así definitivamente el reflujo (9).

La popularidad y generalización alcanzada en el empleo de las técnicas endoscópicas para la resolución del reflujo vesicoureteral ha venido avalada por un flujo constante de publicaciones casi todas ellas favorables a su utilización, que defienden sus múltiples ventajas: mínima agresividad, bajo coste, elevada eficacia, simplicidad de la técnica, posibilidad de repetición si fracasa y rea-

el tratamiento del reflujo vesicoureteral será óptimo cuando 
lización prácticamente ambulatoria $(11,15-17)$. También hay autores, y estamos en parte de acuerdo con ellos, que reflejan la falta de una base científica sólida que avale su efectividad a la hora de ponderar las bondades de esta técnica, pero la heterogeneidad de las muestras en cuanto a número de pacientes y tratamientos aplicados dificulta la aplicación de tratamientos estadísticos concluyentes $(3,18)$. En cualquier caso pensamos, al igual que Vela y cols. (19) y Kirsch y cols. (20), que la técnica debe ser precisa y exacta en cuanto a la localización e inyección de la sustancia para obtener los mejores resultados, y que no está exenta de complicaciones, aunque éstas sean mínimas $(15,21)$.

Desde la introducción, por nuestra parte, del tratamiento endoscópico, hemos podido constatar dos hechos: En primer lugar, ha aumentado el volumen de pacientes con reflujo remitidos por parte de los pediatras para aplicar un tratamiento endoscópico; de otro modo estos pacientes hubieran continuado en sus consultas largo tiempo, al tratarse de reflujos de bajo grado no subsidiarios de cirugía abierta. El tratamiento endoscópico ha permitido solucionar el problema de una forma definitiva sin alargar en exceso la duración del tratamiento médico. Esto ha hecho que aumente la casuística del segundo periodo considerado (2001-2007) y que disminuya significativamente el número de pacientes a los que se les han aplicado técnicas de cirugía abierta respecto al primer periodo (10 vs 24). En segundo lugar, la decisión de pasar de un tratamiento médico a un tratamiento quirúrgico (endoscópico) se ha adelantado en el tiempo; para nuestros pacientes hemos establecido en un año el tiempo de espera para optar por este último si no hay cambios positivos, es decir, si no hay mejoría en los controles cistográficos o existe empeoramiento clínico.

Al igual que sucede en otras series (16), hemos obtenido mejores resultados con el tratamiento endoscópico cuanto menor era el grado del reflujo; también el éxito ha sido superior cuanto menor era la edad de los pacien-

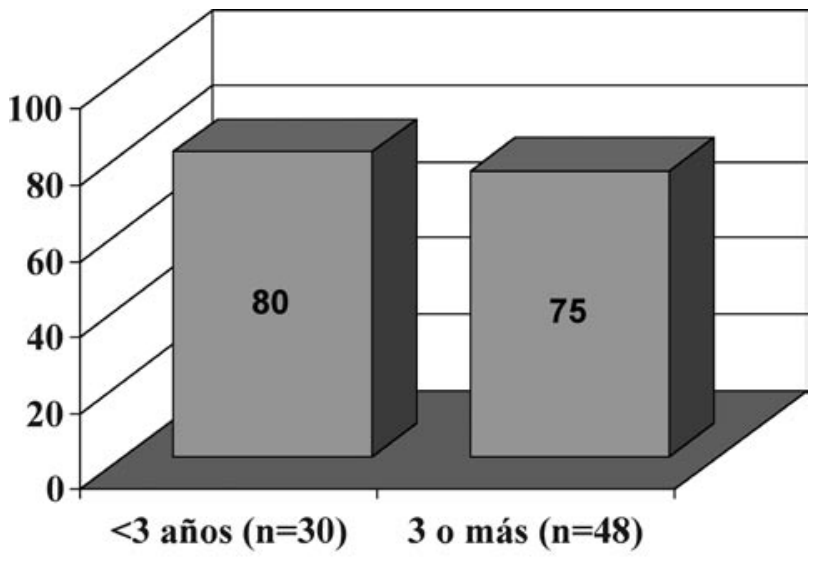

Edad

FIGURA 9. Porción de éxitos con el tratamiento endoscópico (1ํㅡㄹ inyección) según edad (<=3 años). tes. Finalmente, tras la primera inyección, en nuestra serie hemos obtenido mejores resultados en las niñas de forma significativa. Nuestros resultados con el tratamiento endoscópico $(94,9 \%$ de éxitos) son superponibles a los publicados por otros autores en los últimos años (Tabla I) $(4,11,16$, $20,22-25$ ) con tasas de curación que oscilan entre el 63 y el $96 \%$.

En nuestra experiencia, los grados $V$, que casi siempre se han presentado en edades tempranas, se han resuelto de forma precoz mediante la aplicación de cirugía abierta, con una elevada tasa de éxitos en estos reflujos primarios, superior al $95 \%$, por lo que para nosotros es el tratamiento de elección en este grado y en los grado IV que no evolucionen favorablemente con otros tratamientos. De la Peña (3), en su revisión de conjunto, no encuentra evidencias suficientes que aconsejen o desaconsejen cualquiera de los tratamientos en estos grados de reflujo.

Tenemos pues ante nosotros un reto y una responsabilidad ineludible en el manejo de los niños con reflujo vesicoureteral. El buen diagnóstico y el establecimiento de un tratamiento adecuado evitará lesiones irreparables sobre el parénquima renal. Según nuestro criterio, la aplicación de los pilares terapéuticos en el tratamiento del reflujo vesicovereteral sería la siguiente:

\section{1- Observación y seguimiento}

Reservado para casos aislados y siempre en reflujos grado I, en donde existe normalidad anatómica de la unión ureterovesical, normalidad morfológica en la ecografía y en la función renal, sin cuadros febriles y sólo con una infección urinaria en donde el tratamiento médico erradicó este primer brote. Los cultivos de orina, realizados cada 3 meses durante el primer año y cada 6 durante el segundo, deben ser todos negativos. Un control cistográfico al año debe demostrar la desaparición del reflujo o, en todo caso, si éste persiste, no debe aumentar. En el control cistográfico a los 2 años se debe demostrar la desaparición total

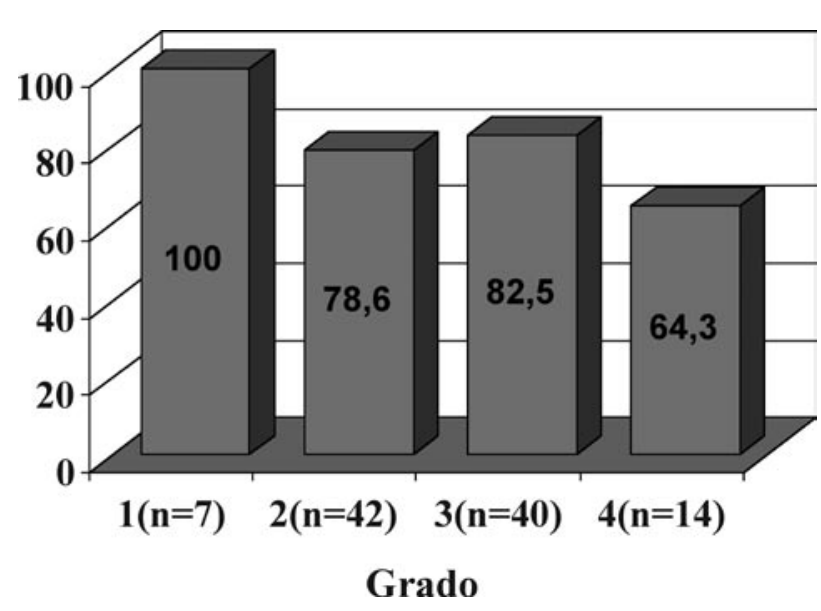

FIGURA 10. Porción de éxitos con el tratamiento endoscópi-

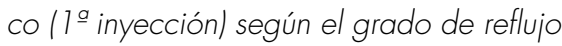


del reflujo. Si esto no ocurriese, se utilizaría un tratamiento médico profiláctico durante un año (punto 2).

\section{2- Tratamiento médico}

Aplicado en todos los reflujos grado I-ll y en los grados III sin clínica febril ni lesión renal parenquimatosa. En todo caso debe existir normalidad anatómica de la unión ureterovesical sin extravesicalización del trayecto submucoso ureteral (comprobado en las placas radiológicas de perfil). La experiencia ha demostrado que en niños menores de 10 años estos reflujos suelen desaparecer cuando se cumplen de forma rígida los esquemas terapéuticos. A nuestro criterio, si estos niños no presentan cuadros febriles, aunque sus cultivos de orina sigan siendo positivos, se debe insistir en el tratamiento profiláctico junto a medidas generales de buena ingesta líquida, evitar el estreñimiento y limpieza correcta de genitales y márgenes anales. Los controles de cultivos de orina son recomendables cada 3 meses el primer año y cada 6 meses los 2 años siguientes. lgualmente, un control cistográfico al año debe demostrar la desaparición o la mejoría del reflujo y el control total de la infección urinaria. De no ser así, deben ser tratados con métodos no invasivos mediante inyección endoscópica submeatal con sustancias biocompatibles. Si en el transcurso del seguimiento presentan más de 2 cuadros febriles con crisis de pielonefritis, sea el grado que sea, deben ser sometidos a tratamiento endoscópico como se ha descrito anteriormente. Este tratamiento médico también se aplicará en casos muy seleccionados de reflujo grado IV, sobre todo en lactantes, aunque adelantando el control cistográfico a los 6-9 meses, siempre que se mantenga estéril la orina, y si no se resuelve se indicará el tratamiento endoscópico.

\section{3- Tratamiento quirúrgico}

Abogamos por el tratamiento quirúrgico en todo reflujo vesicoureteral grado III cuya manifestación clínica sea un cuadro febril con bacteriuria $y$, por supuesto, con cuadro séptico. En estos casos no se debe diferir el tratamiento quirúrgico, por el peligro de daño renal parenquimatoso que deje posteriores secuelas de hipertensión y nefropatía por reflujo. Gracias a los procedimientos endoscópicos actuales, estos tratamientos quirúrgicos no son tan agresivos como los métodos abiertos, siendo por tanto la primera alternativa terapéutica.

En los reflujos grado IV y $\mathrm{V}$ nuestro proceder es siempre quirúrgico, con la salvedad de que los grados IV pueden ser tratados como primera opción con métodos endoscópicos y sólo en caso de fracaso del procedimiento, se realizarán técnicas quirúrgicas abiertas, que empleamos en todos los reflujos grados $V$, por la necesidad no sólo de un seguro control, sino por la posible indicación de modelaje del uréter pélvico que se realizará en función de los hallazgos intraoperatorios.

La posibilidad de desaparición espontánea de los reflujos grado IV y V es, en nuestra experiencia, casi inexistente. No obstante, el Comité Internacional para el Estudio del Reflujo recomienda dejar a criterio del cirujano el empleo de tratamiento médico o quirúrgico en los reflujos grado IV. No creemos oportuno dejar al azar esta decisión, ya que en la actualidad los tratamientos mínimamente invasivos representan, como su propio nombre indica, un mínimo trauma y con sólo este gesto podemos evitar complicaciones y consecuencias que pueden llevar a estos pacientes a situaciones irreversibles.

\section{BIBLIOGRAFIA Y LECTURAS RECOMENDADAS (*lectura de interés $\mathrm{y}^{* *}$ lectura fundamental)}

1. KELALIS, P.P.; KING, L.R.; BELMAN, A.B.: "Clinical Pediatric Urology". $3^{\text {a }}$ ed., W. B. Saunders, Philadelphia, pág 461-462, 1992.

2. MIGUÉLEZ, C.: "Uropediatría. Actualización y controversias". Actas Urol. Esp., 26: 618, 2002.

**3. DE LA PEÑA, E.: "Tratamiento del reflujo vesico-ureteral primario en la infancia: Comparación de dos revisiones sistemáticas". Actas Urol. Esp., 29: 138, 2005.

**4. PURI, P.; PIRKER, M.; MOHANAN, N. y cols.: "Subureteral dextranomer/hyaluronic acid injection as first line treatment in the management of high grade vesicoureteral reflux". J. Urol., 176: 1856, 2006.

5. DWOSKIN, J.Y.; PERLMUTTER, A.D.: "Vesicoureteral reflux in children: A computerized review". J. Urol., 109: 888, 1973.

6. HEIKEL, P.P.; PARKKULAINEN, K.V.: "Vesico-ureteral reflux in children. A classification and results of conservative treatment". Ann. Radil., 9: 37, 1966.

7. REPORT OF THE INTERNATIONAL REFLUX STUDY COMMITTEE: "Medical versus treatment of primary vesicoureteral reflux: A prospective international reflux study in children". J. Urol., 125: 277, 1981.

*8. BERROCAL, T.; GAYÁ, F.; GÓMEZ, N.: "Ecocistografía con contraste: Una nueva modalidad de imagen para diagnosticar el reflujo vesicoureteral". An. Esp. Pediatr., 53: 422, 2000.

*9. MÉNDEZ, R.; SOMOZA, I.; TELLADO, M.G. y cols.: "Reflujo vesicoureteral grados III-IV: Factores implicados en la efectividad del tratamiento endoscópico en pacientes pediátricos". Arch. Esp. Urol., 59: 155, 2006.

10. LYON, R.P.; MARSHALL, S.; TANAGHO, E.A.: "The ureteral orifice: Its configuration and competency". J. Urol., 102: 504, 1969.

*11. MONTERO, M.; MÉNDEZ, R.; TELLADO, M.G. y cols: "Estudio comparativo del tratamiento del reflujo vésico-ureteral en la edad pediátrica: Revisión de una serie de 636 unidades refluyentes". Cir. Pediatr., 12: 144, 1999.

12. RANSLEY, P.G.; RISDON, R.A.: "Reflux and renal scarring”. Brit. J. Radiol., 14: 1978.

13. MENA, E.; DÍAZ, C.; BERNÁ, L.L. y cols.: "Evaluación de lesiones renales mediante 99Tc-DMSA en niños con antecedentes de infección del tracto urinario y su relación con el reflujo vesicoureteral". Rev. Esp. Med. Nucl., 25: 374, 2006.

14. POHL, H.G.; RUSHTON, H.G.: "El diagnóstico y el tratamiento de la infección urinaria en la infancia". AUA Update Series, Medival Trenes, SL ed. pág 1-7, 1999. 
15. SERRANO, A.; BONILLO, M.A.; ESTORNELL, F. y cols.: "Complicaciones del tratamiento endoscópico del reflujo vesicoureteral en la infancia". Actas Urol. Esp., 30: 170, 2006.

16. PELÁEZ, D.; ÁLVAREZ, J.A.: "Estado actual del tratamiento del reflujo vesicoureteral. Análisis de nuestra casuística”. Cir. Pediatr., 14: 112, 2001.

17. CHEN, H.W.; YUAN, S.S.; LIN, C.J.: "Ureteral reimplantation for vesicoureteral reflux: Comparison of minimally invasive extravesical with transvesical and conventional extravesical techniques". Urology, 63: 364, 2004.

18. JIMÉNEZ, C.; ALAMINOS, M.; MARTÍNEZ, L.: "Evidencia científica en el tratamiento endoscópico del reflujo vésico-ureteral". Cir. Pediatr., 15: 122, 2002.

19. VELA, D.; MONTERO, M.; MÉNDEZ, R. y cols.: "Contraindicaciones relatives para el tratamiento endoscópico del reflujo vesicoureteral". Cir. Pediatr., 13:141, 2000.

20. KIRCH, A.J.; PEREZ-BRAYFIELD, M.R.; SCHERZ, H.C.: "Minimally invasive treatment of vesicoureteral reflux with endoscopic injection of dextranomer/hyaluronic acid copolymer: The Children's hospital of Atlanta experience”. J. Urol., 170: 211, 2003.

21. VANDERSTEEN, D.R.; ROUTH, J.C.; KIRCH, A.J. y cols.: "Postoperative ueteral obstruction after subureteral injection of dextranomer/hyaluronic Acid copolymer". J. Urol., 176: 1593, 2006.

**22. CAPOZZA, N.; LAIS, A.; NAPPO, S. y cols.: "The role of endoscopic treatment of vesicoureteral reflux: A 17-Year experience". J. Urol., 172: 1626, 2004.

23. SUGIYAMA, T.; HANAI, T.; HASHIMOTO, K. y cols.: "Long-term outcome of the endoscopic correction of vesico-ureteric reflux: A comparison of injected substances". BJU Int., 94: 381, 2004.

24. AL-HUNAYAN, A.A.; KEHINDE, E.O.; ELSALAM, M.A. y cols.: "Outcome of endoscopic treatment for vesicoureteral reflux in children using polydimethylsiloxane”. J. Urol., 168: 2181, 2002.

25. HERZ, D.; HAFEZ, A.; BAGLI, D. y cols.: "Efficacy of endoscopic subureteral polydimethylsiloxane injection for treatment of vesicoureteral reflux in children: A North American clinical report". J. Urol., 166: 1880, 2001. 